\title{
Commande sous optimale par retour de sortie pour le suivi de trajectoires des modèles TS incertains
}

\author{
Kevin Guelton*_ Noureddine Manamanni* \\ Alexandre Kruszewski**_ Thierry-Marie Guerra*** \\ Badr Mansouri*
}

* CReSTIC EA3804, Université de Reims Champagne-Ardenne

Moulin de la Housse BP 1039, F-51687, Reims Cedex 2

\{prénom,nom\}@univ-reims.fr

** LAGIS FRE CNRS 3303, Ecole Centrale de Lille

Avenue Paul Langevin, BP 48, F-59651, Villeneuve d'Ascq Cedex

\{prénom,nom\}@univ-valenciennes.fr

*** LAMIH FRE CNRS 3304, Université de Valenciennes et du Hainaut Cambrésis

Le Mont Houy, F-59313, Valenciennes Cedex 9

\{prénom,nom\}@univ-valenciennes.fr

RÉSUMÉ. Ce travail traite de la commande en suivi de trajectoires par retour de sortie pour les systèmes dynamiques non linéaires incertains décrits par des multi-modèles de type Takagi-Sugueno (TS). La synthèse de la loi de commande et de l'observateur est basée sur un critère quadratique relatif à l'erreur de poursuite. Les conditions sont alors écrites sous forme de LMI (Linear Matrix Inequality) par le biais des propriétés de l'ISS (Input to State Stability). Un exemple de suivi de trajectoire d'un simple pendule inversé mis en mouvement par un chariot mobile illustre l'approche proposée.

ABSTRACT. In this paper, the design of output feedback tracking controllers is addressed for uncertain non linear dynamic system using TS fuzzy model. A control scheme, based on a quadratic criterion related to the tracking error, a reference model and a fuzzy observer, is proposed. The main result of this paper is to provide Linear Matrix Inequality (LMI) based conditions through the ISS (Input to State Stability) properties. The design of a tracking controller for an inverted pendulum on a cart is proposed to illustrate the proposed approach.

MOTS-CLÉS : Poursuite de trajectoires, modèles Takagi Sugeno, systèmes non linéaires, LMI, commande optimale.

KEYWORDS: Tracking control, Takagi-Sugeno models, non linear systems, LMI, optimal control.

RS - JESA. Volume $\mathrm{X}-\mathrm{n}^{\circ} \mathrm{x}$ /année, Commande robuste et applications, pages 1 à 24 


\section{Introduction}

La synthèse de lois de commande pour les systèmes non linéaires incertains demeure une problématique d'actualité en automatique. Parmi les approches théoriques dévolues à la commande de ces systèmes, l'utilisation de la classe des multi-modèles flous de type Takagi-Sugeno (TS) suscite un intérêt croissant. En effet, celle-ci permet de représenter un système non linéaire par un ensemble de polytopes interconnectés par des fonctions non linéaires (Takagi et Sugeno, 1985; Tanaka et Wang, 2001). Afin de stabiliser ce type de systèmes, les lois de commandes dites PDC (Parallel Distributed Compensation) sont communément utilisées (Wang et al., 1996). L'intérêt majeur de ces contrôleurs est qu'ils possèdent la même structure d'interconnections que les multi-modèles à partir desquels ils sont synthétisés. Cette particularité permet alors d'étendre, pour la classe de systèmes non linéaires considérée, certaines théories issues de la commande des systèmes linéaires (Tanaka et Wang, 2001).

De nombreux travaux traitent de l'analyse de la stabilité, de la stabilisation et de l'observation des modèles TS (Tanaka et Wang, 2001; Sala et al., 2005; et références incluses). Ceux-ci sont essentiellement basés sur la seconde méthode de Lyapunov de manière à aboutir, lorsque cela est possible, à la formulation d'un problème d'optimisation convexe sous forme d'Inégalités Linéaires Matricielles (LMI) (Boyd et al., 1994). La stabilisation par retour d'état a été abordée pour plusieurs classes de modèles TS. Par exemple, les modèles avec incertitudes paramétriques bornées (Chen et al., 2000 ; Park, 2004 ; Tanaka et al., 1996), avec retard (Cao et Frank, 2001 ; Xu et Lam, 2005), etc. Des critères de performances tels que le placement de pôles des modèles locaux (Hong et Langari, 2000), l'introduction de performances $H_{2}, H_{\infty}$ (Zerar et al., 2008), le critère du cercle (Ban et Gao, 2007), ou encore la commande optimale (Wu et Lin, 2000, Tanaka et Wang, 2001, Park et al., 2004). Notons que la commande optimale est une des approches les plus élaborées pour spécifier les performances d'un système (Alazard et al., 1999).

Lorsque l'état du système n'est pas (ou partiellement) disponible, la stabilisation par retour de sortie peut être envisagée. Dans ce cadre, trois approches sont communément considérées. Les deux premières consistent à synthétiser des contrôleurs statiques (Chadli et al., 2002 ; Huang et Nguang, 2007 ; Bouarar et al., 2009) ou dynamiques (Assawinchaichote et al., 2004 ; Li et al., 2000 ; Zerar et al., 2008 ; Guelton et al., 2009) par retour de sortie. L'implémentation d'un contrôleur statique s'avère relativement simple et permet de minimiser le coût de calcul en ligne (Syrmos et al., 1997). Néanmoins, cette simplicité s'acquière au prix d'un compromis en termes de performances souhaité en boucle fermée. La commande dynamique permet, quant à elle, d'améliorer les performances en introduisant des spécifications relatives à la dynamique souhaitée en boucle fermée (Chen et al., 2000 ; Zerar et al., 2008). Néanmoins, ce type d'approche s'avère conservatif, voire 
restrictif, pour les systèmes TS dans la mesure où l'expression de la dynamique en boucle fermée conduit à l'apparition de nombreux termes croisés au sein des conditions LMI (Guelton et al., 2009). Une dernière approche de commande par retour de sortie, permettant un compromis entre implémentation et performances, consiste à introduire un observateur afin d'estimer les variables d'états non mesurées sur la base des mesures d'entrées-sorties (Tanaka et al., 1998; Ma et al., 1998 ; Yoneyama et al., 2000; Liu et Zang, 2003 ; Guerra et al., 2006).

Malgré une littérature abondante concernant la stabilisation des modèles TS, la synthèse de lois de commande robuste par retour de sortie, garantissant le suivi de trajectoires, reste à ce jour peu traité. Tseng et Chen ont considéré le problème de suivi de trajectoires pour les modèles TS soumis à des perturbations externes (Tseng et Chen, 2001). Cette approche est basée sur l'introduction d'un modèle de référence et d'un critère $H_{\infty}$ afin de minimiser l'erreur de poursuite. Les conditions obtenues ont été proposées sous forme BMI (Bilinear Matrix Inequality). De plus, dans le cas incertain, une approche similaire a été proposée (Tong et al., 2002) mais au détriment de la confusion entre l'erreur d'observation et l'erreur de poursuite menant à des conditions BMI inapplicables (Chadli et El Hajjaji, 2006). Plus récemment, afin de palier ce problème, des conditions LMI pour la synthèse robuste de lois de commande par retour de sortie en suivi de trajectoires pour les systèmes TS incertains et perturbés ont été proposées (Mansouri et al., 2009). Néanmoins, le caractère optimal de ces approches n'est pas établi. En effet, à notre connaissance, il n'existe pas, à ce jour, dans la littérature, de résultat sous forme LMI permettant la synthèse de lois de commande optimales pour le suivi de trajectoires des systèmes non linéaires décrit par des modèles TS avec incertitudes paramétriques. Ceci motive donc la présente étude.

Dans cet article, des conditions LMI pour la synthèse de lois de commande robuste par retour de sortie, garantissant le suivi de trajectoires, pour les modèles TS incertains sont proposées. Dans un premier temps, les modèles TS incertains ainsi que leurs techniques d'obtention sont présentés. Ensuite, une structure de commande par retour de sortie, permettant de réaliser le suivi de trajectoires, basée sur l'introduction d'un observateur et d'un modèle de référence, est présentée. Afin de réaliser la synthèse optimale des gains de commande et d'observateur, des conditions LMI sont proposées. Celles-ci permettent la minimisation d'une fonction de coût relative à l'erreur de poursuite et sont obtenues par le biais des propriétés de l'ISS (Input to State Stability) (Sontag et Wang, 1995). Enfin, un exemple de suivi de trajectoire d'un simple pendule inversé mis en mouvement par un chariot mobile est considéré afin d'illustrer l'approche de synthèse proposée.

\section{Classe de modèles considérée et obtention des modèles Takagi-Sugeno}

Historiquement, lors de leur introduction en 1985, les modèles flous de type TS sont décrits par un ensemble de $r$ règles floues Si-Alors (Takagi et Sugeno, 1985). 
Leur caractéristique principale est de permettre de représenter localement les relations entrées-sorties d'un système en exprimant chaque conclusion par un modèle local. Dans cet article, nous nous intéressons à la classe des modèles flous de type TS avec incertitudes paramétriques dont la $i^{\text {ème }}$ règle s'écrit:

$$
\begin{aligned}
& \text { Si } z_{1}(t) \text { est } F_{i 1} \text { et } \ldots \text { et } z_{j}(t) \text { est } F_{i j} \text { et } \ldots \text { et } z_{p}(t) \text { est } F_{i p} \\
& \text { Alors }\left\{\begin{array}{l}
\dot{x}(t)=\left(A_{i}+\Delta A_{i}(t)\right) x(t)+\left(B_{i}+\Delta B_{i}(t)\right) u(t) \\
y(t)=C_{i} x(t)
\end{array}\right.
\end{aligned}
$$

où, pour $i=1, \ldots, r$ et $j=1, \ldots, p, F_{i j}$ sont les sous-ensembles flous réalisant une partition exacte de l'univers du discourt, $x(t) \in \mathbb{R}^{n}$ est le vecteur d'état, $u(t) \in \mathbb{R}^{m}$ est le vecteur de commande, $y(t) \in \mathbb{R}^{q}$ est le vecteur de sortie du système et $z_{j}(t)$ sont les $p$ variables de prémisses dépendantes des variables d'état et/ou des grandeurs mesurables du système. $A_{i} \in \mathbb{R}^{n \times n}, \quad B_{i} \in \mathbb{R}^{n \times m}$ et $C_{i} \in \mathbb{R}^{q \times n}$ sont respectivement la matrice d'état, la matrice d'entrée et la matrice de sortie du modèle nominal associé à la $i^{\text {ème }}$ règle. Pour cette même règle, les matrices $\Delta A_{i}(t)$ et $\Delta B_{i}(t)$ représentent des incertitudes paramétriques bornées dont les composantes peuvent être normalisées et sont, le cas échéant, variables dans le temps (Zhou et Kargonekar, 1988), telles que:

$$
\Delta A_{i}(t)=H_{i} \Delta a(t) E_{a i} \text { et } \Delta B_{i}(t)=H_{i} \Delta b(t) E_{b i}
$$

où $\Delta a^{T}(t) \Delta a(t) \leq I, \Delta b^{T}(t) \Delta b(t) \leq I$ et $H_{i}, E_{a i}, E_{b i}$ et $E_{c i}$ sont des matrices à coefficients constants.

Soient $F_{i j}\left(z_{i}(t)\right)$, les valeurs d'appartenances de $z_{j}(t)$ dans les sous-ensembles flous $F_{i j}$. La défuzzification barycentrique de [1] s'obtient en posant :

$$
w_{i}(z(t))=\prod_{j=1}^{p} F_{i j}\left(z_{j}(t)\right)>0
$$

et en considérant les fonctions d'appartenances :

$$
h_{i}(z(t))=\frac{w_{i}(z(t))}{\sum_{i=1}^{r} w_{i}(z(t))}
$$

qui possèdent la propriété d'une somme convexe, i.e. : 


$$
\forall t \geq 0, \text { pour } i=1, \ldots, r, h_{i}(z(t)) \geq 0 \text { et } \sum_{i=1}^{r} h_{i}(z(t))=1
$$

où $z(t)=\left[\begin{array}{llll}z_{1}(t) & z_{2}(t) & \ldots & z_{p}(t)\end{array}\right]$,

Ainsi, l'inférence des $r$ règles floues [1] conduit à l'obtention d'un multi-modèle de type TS donné par la représentation d'état non linéaire suivante:

$$
\left\{\begin{array}{l}
\dot{x}(t)=\sum_{i=1}^{r} h_{i}(z(t))\left(\left(A_{i}+\Delta A_{i}(t)\right) x(t)+\left(B_{i}+\Delta B_{i}(t)\right) u(t)\right) \\
y(t)=\sum_{i=1}^{r} h_{i}(z(t)) C_{i} x(t)
\end{array}\right.
$$

Remarque 1: La représentation d'état [6] sous forme TS constitue une modélisation polytopique convexe assimilable à un modèle Quasi-LPV (Linéaire à Paramètres Variants). Notons qu'à la différence des systèmes LPV, dont les paramètres varient directement par rapport au temps, les fonctions d'activation $h_{i}(z(t))$ des modèles Quasi-LPV peuvent dépendre de l'état, de l'entrée ou de toutes autres variables de décision $z(t)$ du système.

Dans la littérature, trois approches sont couramment décrites pour l'obtention d'un modèle TS :

- Identification des modèles TS à partir de mesures acquises des entrées et sorties d'un système physique (Gasso et al., 1999; Gasso, 2000). Dans ce cas, le problème d'identification du modèle non linéaire se réduit à l'identification de modèles locaux autour de points de fonctionnement préalablement choisis d'un système non linéaire. Cette méthode est utilisée dans le cas de systèmes dotés d'une dynamique difficile à décrire à l'aide d'un modèle analytique.

- Linéarisation d'un modèle non linéaire autour d'un ensemble fini de points de fonctionnement (Ma et al., 1998 ; Tanaka et Wang 2001). Un représentant TS est obtenu par l'interconnexion des dynamiques locales à l'aide de fonctions d'appartenance judicieusement choisies (gaussiennes, triangulaires, trapézoïdales, etc.). Notons que cette approche constitue dans la plupart des cas une approximation du modèle non linéaire considéré.

- Décomposition en secteurs non linéaires basée sur des transformations polytopiques convexes des termes non linéaires d'un système dynamique (Kawamoto et al., 1992 ; Tanaka et Wang, 2001 ; Morère, 2001). Cette méthode garantie la construction d'un modèle TS représentant exactement un modèle non linéaire sur un espace compact de ses variables d'état. 
Dans la suite, nous considèrerons la troisième méthode dans la mesure où elle présente l'avantage de s'affranchir d'approximations de modélisation lors du passage du modèle analytique non linéaire au modèle TS (hormis une réduction éventuelle de l'espace de validité du modèle TS). Par ailleurs, notons que de manière générale, les transformations polytopiques convexes sont injectives. De ce fait, une infinité de modèles TS peuvent être associés à un système non linéaire donné. Néanmoins, une approche systématique d'obtention des modèles TS à partir d'un système non linéaire affine en la commande peut être employée. Celle-ci est résumée par le lemme suivant.

Lemme 1 (Morère, 2001; Tanaka et Wang, 2001): Soit $f(x(t)): \mathbb{R} \rightarrow \Omega$ une fonction bornée avec $\Omega$ un sous-espace compact de $\mathbb{R}$. Il existe toujours deux fonctions $w_{1}(x(t)) \geq 0$ et $w_{2}(x(t)) \geq 0$ convexes $\left(w_{1}+w_{2}=1\right)$ ainsi que, deux scalaires $\alpha$ et $\beta$ tels que $f(x(t))=\alpha \times w_{1}(x(t))+\beta \times w_{2}(x(t))$.

Preuve: Sous l'hypothèse que la fonction $f(x(t))$ est bornée telle que $\alpha \leq f(x(t)) \leq \beta$, le lemme 1 est vérifié avec, par exemple, $\alpha=\max (f(x(t)))$, $\beta=\min (f(x(t))), w_{1}(x(t))=\frac{f(x(t))-\beta}{\alpha-\beta}$ et $w_{2}(x(t))=\frac{\alpha-f(x(t))}{\alpha-\beta}$.

Notons enfin que, selon les propriétés des termes non linéaires rencontrés dans le modèle de connaissance non linéaire, on distingue deux types de représentants TS. En effet :

- Si toutes les non-linéarités du système sont continues et bornées, alors le modèle TS représente de manière exacte le système non linéaire sur l'intégralité de l'espace des variables d'état. Dans ce cas, le découpage en secteur non linéaire est dit « global » (cf. figure 1).

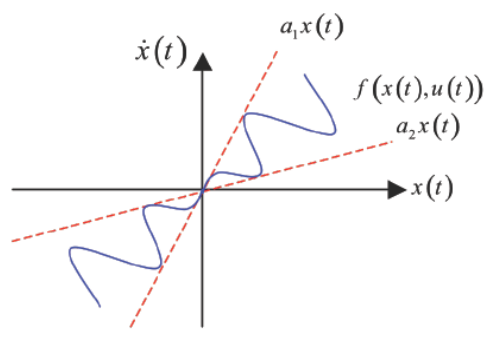

Figure 1 - Secteur non linéaire global.

- si tout ou partie des non-linéarités du système sont continues mais non bornées, alors le modèle TS représente de façon exacte le système non linéaire sur un 
sous-espace compact de l'espace des variables d'état. Dans ce cas, le découpage en secteur non linéaire est dit « local» (cf. figure 2).

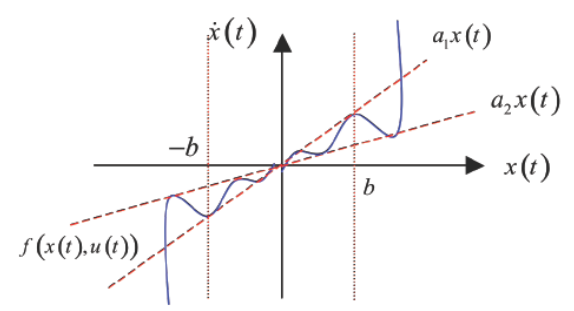

Figure 2 - Secteur non linéaire local.

Exemple 1 : Soit le système non linéaire autonome :

$$
\dot{x}(t)=x(t) \cos (x(t))
$$

La fonction $\cos (x(t))$ est continue et bornée sur $[-1,1]$. D'après le lemme 1 , on peut écrire de manière exacte:

$$
\cos (x(t))=\underbrace{\frac{\cos (x(t))+1}{2}}_{h_{1}(x(t))} \times 1+\underbrace{\frac{1-\cos (x(t))}{2}}_{h_{2}(x(t))} \times(-1)
$$

D'où le représentant TS de [7] :

$$
\dot{x}(t)=\sum_{i=1}^{2} h_{i}(x(t)) a_{i} x(t)
$$

avec $a_{1}=1$ et $a_{2}=-1$.

Remarque 2: Le nombre de règles d'un modèle TS obtenu via une décomposition en secteurs non linéaires dépend directement du nombre de non-linéarités à découper. Ainsi, lorsque l'on a $n_{l}$ termes non linéaires pour un système dynamique donné, un représentant TS obtenu par décomposition en secteurs non linéaires est constitué de $2^{n_{l}}$ règles floues. 


\section{Structure de commande par retour de sortie en suivi de trajectoires}

Le schéma de la figure 3 présente la structure de commande adoptée. Celle-ci repose sur l'emploi d'un observateur TS pour assurer le retour de sortie et d'un modèle de référence permettant le suivi de trajectoires (Mansouri et al., 2009).

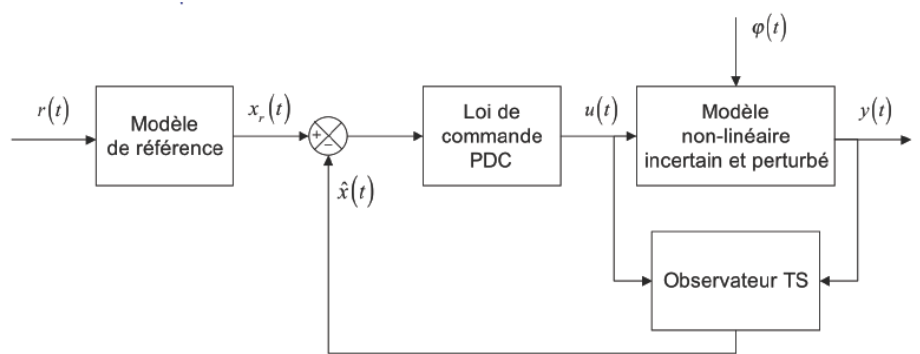

Figure 3 - Structure de commande en suivi de trajectoires par retour de sortie.

Pour des raisons de commodité, nous supposons que les variables de prémisses sont mesurables, i.e. $\hat{z}(t)=z(t)$ (Tanaka et Wang, 2001). Un observateur TS peut être obtenu à partir de la même structure d'interconnexion que le modèle TS à partir duquel il est synthétisé. Ainsi, en considérant un modèle TS de la forme [6], l'observateur est donné par :

$$
\left\{\begin{array}{l}
\dot{\hat{x}}(t)=\sum_{i=1}^{r} h_{i}(z(t))\left(A_{i} \hat{x}(t)+B_{i} u(t)+L_{i}(y(t)-\hat{y}(t))\right) \\
\hat{y}(t)=\sum_{i=1}^{r} h_{i}(z(t)) C_{i} \hat{x}(t)
\end{array}\right.
$$

où $\hat{x}(t) \in \mathbb{R}^{n}$ est le vecteur d'état estimé et les matrices $L_{i}$, pour $i=1, \ldots, r$, sont les gains de l'observateur à synthétiser.

Afin d'assurer le suivi de trajectoires, nous considérons un modèle de référence dont la structure est donnée par (Tseng et al., 2001):

$$
\dot{x}_{r}(t)=A_{r} x_{r}(t)+r(t)
$$

où $x_{r}(t)$ est l'état de référence, $A_{r}$ est une matrice Hurwitz et $r(t)$ est l'entrée de consigne.

Considérons maintenant la loi de commande par Compensations Parallèles Distribuées (PDC) donnée par : 


$$
u(t)=-\sum_{i=1}^{r} h_{i}(z(t)) K_{i}\left(x_{r}(t)-\hat{x}(t)\right)
$$

où les matrices $K_{i}$, pour $i=1, \ldots, r$, sont les gains de commande à synthétiser.

Soit $e_{o}(t)=x(t)-\hat{x}(t)$ et $e_{p}(t)=x(t)-x_{r}(t)$, respectivement les erreurs d'observation et de poursuite. En considérant la loi de commande [12], le système [6], l'observateur [10] et le modèle de référence [11], on peut écrire :

$$
\begin{aligned}
\dot{x}(t) & =\sum_{i=1}^{r} \sum_{j=1}^{r} h_{i}(z(t)) h_{j}(z(t))\left(-\left(B_{i} K_{j}+\Delta B_{i} K_{j}\right) e_{o}(t)\right. \\
& \left.+\left(A_{i}+B_{i} K_{j}+\Delta A_{i}+\Delta B_{i} K_{j}\right) e_{p}(t)+\left(A_{i}+\Delta A_{i}\right) x_{r}(t)\right)
\end{aligned}
$$

De plus, la dynamique de l'erreur d'estimation peut être exprimée par :

$$
\begin{aligned}
\dot{e}_{o}(t) & =\sum_{i=1}^{r} \sum_{j=1}^{r} h_{i}(z(t)) h_{j}(z(t))\left(\left(A_{i}-L_{i} C_{j}-\Delta B_{i} K_{j}\right) e_{o}(t)\right. \\
& \left.+\left(\Delta A_{i}+\Delta B_{i} K_{j}\right) e_{p}(t)+\Delta A_{i} x_{r}(t)\right)
\end{aligned}
$$

ainsi que la dynamique de l'erreur de poursuite, par :

$$
\begin{aligned}
\dot{e}_{p}(t) & =\sum_{i=1}^{r} \sum_{j=1}^{r} h_{i}(z(t)) h_{j}(z(t))\left(-\left(B_{i} K_{j}+\Delta B_{i} K_{j}\right) e_{o}(t)\right. \\
& \left.+\left(A_{i}+B_{i} K_{j}+\Delta A_{i}+\Delta B_{i} K_{j}\right) e_{p}(t)+\left(A_{i}-A_{r}+\Delta A_{i}\right) x_{r}(t)\right)-r(t)
\end{aligned}
$$

Finalement, en considérant le vecteur d'état augmenté $\tilde{x}(t)=\left[\begin{array}{lll}e_{o}(t) & e_{p}(t) & x_{r}(t)\end{array}\right]^{T}$, on aboutie au système augmenté décrivant la dynamique en boucle fermée donné par :

$$
\dot{\tilde{x}}(t)=\sum_{i=1}^{r} \sum_{j=1}^{r} h_{i}(z(t)) h_{j}(z(t)) \tilde{A}_{i j} \tilde{x}(t)+\tilde{\theta}(t)
$$

avec $\tilde{A}_{i j}=\left[\begin{array}{ccc}A_{i}-L_{i} C_{j}-\Delta B_{i} K_{j} & \Delta A_{i}+\Delta B_{i} K_{j} & \Delta A_{i} \\ -B_{i} K_{j}-\Delta B_{i} K_{j} & A_{i}+B_{i} K_{j}+\Delta A_{i}+\Delta B_{i} K_{j} & A_{i}-A_{r}+\Delta A_{i} \\ 0 & 0 & A_{r}\end{array}\right]$ et $\tilde{\theta}(t)=\left[\begin{array}{lll}0 & -r^{T}(t) & r^{T}(t)\end{array}\right]^{T}$. 
L'objectif est, maintenant, de synthétiser les gains $K_{i}$ et $L_{i}$ qui assureront la stabilité du modèle TS augmenté [16] et qui, par conséquent, garantiront le suivi de trajectoires par retour de sortie du modèle TS [6]. Dans ce dessein, des conditions écrites sous forme d'Inégalités Linéaires Matricielles (LMI) sont proposées à la section suivante.

\section{Conditions LMI pour la synthèse d'une loi de commande sous optimale}

Afin de synthétiser une loi de commande optimale, la minimisation d'une fonction de coût relative à l'erreur de poursuite $e_{p}(t)$ et au vecteur de commande $u(t)$ peut être considérée. Cette fonction est donnée par le critère quadratique suivant:

$$
J=\int_{0}^{\infty}\left(e_{p}^{T}(t) Q e_{p}(t)+u^{T}(t) R u(t)\right) d t
$$

où $Q$ et $R$ sont des matrices définies positives de dimensions appropriées.

Cette fonction de coût peut être exprimée par rapport à l'état augmenté $\tilde{x}(t)$ telle que :

$$
J=\int_{0}^{\infty}\left(\tilde{x}^{T}(t) \tilde{Q} \tilde{x}(t)+u^{T}(t) R u(t)\right) d t
$$

$\operatorname{avec} \tilde{Q}=\operatorname{diag}\left[\begin{array}{lll}0 & Q & 0\end{array}\right]$.

Afin d'aboutir à des conditions LMI, nous exploiterons les lemmes et propriétés matricielles usuels suivants.

Lemme 1 (Zhou et Khargonekar, 1988): Soient deux matrices $A$ et $B$ de dimensions appropriées, il existe un scalaire positif $\mu$ tel que :

$$
A^{T} B+B^{T} A \leq \mu A^{T} A+\mu^{-1} B^{T} B
$$

Lemme 2 (Guerra et al., 2006) : Soit une matrice définie négative $\mho<0$, pour toutes matrices $X$ de dimensions appropriées, on a $X^{T} \mho X<0$ et :

$$
\exists \varepsilon \in \mathfrak{R}, \varepsilon>0 \text { et } X^{T} \mho X \leq-\varepsilon\left(X+X^{T}\right)-\varepsilon^{2} \mho^{-1}
$$

Propriété 1 : Pour une matrice $R>0$ de dimension appropriée, l'inégalité suivante est vérifiée : 


$$
K_{i}^{T} R K_{j}+K_{j}^{T} R K_{i} \leq K_{i}^{T} R K_{i}+K_{j}^{T} R K_{j}
$$

Le théorème suivant résume les conditions, formulées en termes de LMI, permettant de garantir la stabilité du système augmenté [16] et, par conséquent les performances en suivi de trajectoires spécifiées sous la contrainte [18] (Mansouri et al., 2007).

Théorème 1: Le système en boucle fermée [16] est globalement asymptotiquement stable et minimise la fonction de coût [18] avec un taux de décroissance $\alpha$, s'il existe des matrices $P_{1}=P_{1}^{T}>0, P_{3}=P_{3}^{T}>0, N=N^{T}>0, Z_{i}, Y_{i}, \bar{Q}=Q^{-1}>0$, $\bar{R}=R^{-1}>0$ et des scalaires positifs $\lambda, \varepsilon, \mu_{1}, \mu_{2}, \mu_{3}, \mu_{4}, \mu_{5}, \mu_{6}, \mu_{7}$ et $\mu_{8}$, telles que [22] et [23] soient vérifiées, pour tout $i=1, \ldots, r$ et $j=1, \ldots, r$.

$\min \lambda$ sous les contraintes:

$$
\begin{aligned}
& {\left[\begin{array}{cc}
\lambda-e_{o}{ }^{T}(0) P_{1} e_{o}(0)-x_{r}{ }^{T}(0) P_{3} x_{r}(0) & e_{p}{ }^{T}(0) \\
e_{p}(0) & N
\end{array}\right]>0}
\end{aligned}
$$

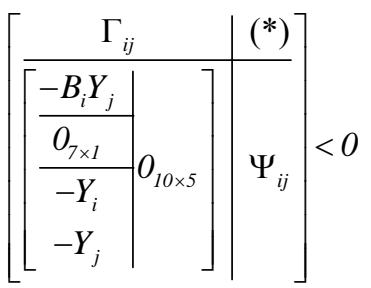

$$
\text { avec } \Gamma_{i j}=\left[\begin{array}{cccccc}
-2 \varepsilon N & (*) & (*) & (*) & 0 & 0 \\
E_{b i} Y_{j} & -\mu_{1} I & 0 & 0 & 0 & 0 \\
E_{b i} Y_{j} & 0 & -\mu_{5} I & 0 & 0 & 0 \\
\varepsilon I & 0 & 0 & \Gamma_{i j}(4,4) & (*) & (*) \\
0 & 0 & 0 & H_{i}^{T} P_{1} & -\left(\mu_{1}+\mu_{2}+\mu_{3}\right)^{-1} I & 0 \\
0 & 0 & 0 & H_{i}^{T} P_{1} & 0 & -\mu_{4} I
\end{array}\right] \text {, }
$$


12 RS - JESA. Volume $\mathrm{X}-\mathrm{n}^{\circ} \mathrm{x} /$ année. Commande robuste et applications

$\Psi_{i j}=\left[\begin{array}{cccccccccc}\Psi_{i j}(1,1) & (*) & (*) & (*) & (*) & (*) & (*) & 0 & (*) & (*) \\ N & -\bar{Q} & 0 & 0 & 0 & 0 & 0 & 0 & 0 & 0 \\ E_{b i} Y_{j} & 0 & -\mu_{2} I & 0 & 0 & 0 & 0 & 0 & 0 & 0 \\ E_{b i} Y_{j} & 0 & 0 & -\mu_{7} I & 0 & 0 & 0 & 0 & 0 & 0 \\ E_{a i} N & 0 & 0 & 0 & -\mu_{3} I & 0 & 0 & 0 & 0 & 0 \\ E_{a i} N & 0 & 0 & 0 & 0 & -\mu_{6} I & 0 & 0 & 0 & 0 \\ \left(A_{i}^{T}-A_{r}^{T}\right) & 0 & 0 & 0 & 0 & 0 & \Psi_{i j}(7,7) & (*) & 0 & 0 \\ 0 & 0 & 0 & 0 & 0 & 0 & E_{a i} & -\mu_{8} & 0 & 0 \\ Y_{i} & 0 & 0 & 0 & 0 & 0 & 0 & 0 & -2 \bar{R} & 0 \\ Y_{j} & 0 & 0 & 0 & 0 & 0 & 0 & 0 & 0 & -2 \bar{R}\end{array}\right]$,

$\Gamma_{i j}(4,4)=P_{1} A_{i}+A_{i}^{T} P_{1}-Z_{i} C_{j}-C_{j}^{T} Z_{i}^{T}+2 \alpha P_{1}$,

$\Psi_{i j}(1,1)=A_{i} N+B_{i} Y_{j}+(*)+2 \alpha N+\left(\mu_{5}+\mu_{6}+\mu_{7}+\mu_{8}\right) H_{i} H_{i}^{T}$

et $\Psi_{i j}(7,7)=A_{r}{ }^{T} P_{3}+P_{3} A_{r}+2 \alpha P_{3}+\mu_{4} E_{a i}{ }^{T} E_{a i}$.

Enfin, la synthèse des gains de commande et d'observation s'obtient en considérant $K_{i}=Y_{i} N^{-1}$ et $L_{i}=Z_{i} P_{1}^{-1}$ pour $i=1, \ldots, r$.

Preuve: Soit la fonction candidate de Lyapunov quadratique :

$$
V(\tilde{x}(t))=\tilde{x}^{T}(t) \tilde{P} \tilde{x}(t)
$$

On note que, pour $\tilde{x}(0)=0$ on a $V(\tilde{x}(t))=0$. Le système en boucle fermée [16] est quadratiquement stable si, $\forall \tilde{x}(0) \neq 0, V(\tilde{x}(t))>0$, i.e. $\tilde{P}=\tilde{P}^{T}>0$, et $\dot{V}(\tilde{x}(t))<0$. L'obtention d'une solution optimale au regard du critère quadratique [18] nécessite de vérifier l'inégalité suivante:

$$
\dot{V}(\tilde{x}(t))+\tilde{x}^{T}(t) \tilde{Q} \tilde{x}(t)+u^{T}(t) R u(t)<0
$$

En intégrant [25] on peut écrire :

$$
\left.V(\tilde{x}(t))\right|_{0} ^{\infty}+\int_{0}^{\infty}\left(\tilde{x}^{T}(t) \tilde{Q} \tilde{x}(t)+u^{T}(t) R u(t)\right) d t<0
$$

Que l'on peut réécrire sous la forme :

$$
\int_{0}^{\infty}\left(\tilde{x}^{T}(t) \tilde{Q} \tilde{x}(t)+u^{T}(t) R u(t)\right) d t<\tilde{x}^{T}(0) \tilde{P} \tilde{x}(0)
$$

Notons que si l'inégalité [25] est vérifiée, on parle alors de solution sous optimale puisque la borne supérieure de [18] s'exprime en fonction des conditions 
initiales de la fonction candidate de Lyapunov, i.e. du terme $\tilde{X}^{T}(0) \tilde{P} \tilde{x}(0)$. Afin d'aboutir à la condition LMI [22], on introduit un paramètre $\lambda>0$ à minimiser tel que :

$$
J \leq \tilde{X}^{T}(0) \tilde{P} \tilde{x}(0) \leq \lambda
$$

On pose $\tilde{P}=\left[\begin{array}{ccc}P_{1} & 0 & 0 \\ 0 & P_{2} & 0 \\ 0 & 0 & P_{3}\end{array}\right]$ avec $P_{1}=P_{1}^{T}>0, \quad P_{2}=P_{2}^{T}>0$ et $P_{3}=P_{3}^{T}>0$. L’inégalité [28] peut être réécrite sous la forme :

$$
\lambda-e_{o}^{T}(0) P_{1} e_{o}(0)-x_{r}^{T}(0) P_{3} x_{r}(0)-e_{p}^{T}(0) P_{2} e_{p}(0)>0
$$

En considérant le changement de variable $N=P_{2}^{-1}$ et en appliquant le complément de Schur, on obtient l'inégalité [22].

De plus, afin d'améliorer les performances, on introduit un taux de décroissance $\alpha>0$ de la fonction de Lyapunov [24]. De ce fait, l'inégalité [25] est vérifiée si:

$$
\dot{V}(\tilde{x}(t))+2 \alpha V(\tilde{x}(t))+\tilde{x}^{T}(t) \tilde{Q} \tilde{x}(t)+u^{T}(t) R u(t)<0
$$

Sachant que $x_{r}(t)-\hat{x}(t)=e_{o}(t)-e_{p}(t)$, on peut réécrire la loi de commande [12] telle que :

$$
u(t)=\sum_{i=1}^{r} h_{i}(z(t)) \tilde{K}_{i} \tilde{x}(t)
$$

avec $\tilde{K}_{i}=\left[\begin{array}{lll}-K_{i} & K_{i} & 0\end{array}\right]$.

En considérant la dynamique en boucle fermée [16] et l'écriture augmentée de la loi de commande [31], l'inégalité [30] peut être réécrite sous la forme :

$$
\begin{aligned}
& \sum_{i=1}^{r} \sum_{j=1}^{r} h_{i}(z(t)) h_{j}(z(t))\left(\tilde{x}^{T}(t)\left(\tilde{A}_{i j}^{T} \tilde{P}+\tilde{P} \tilde{A}_{i j}+2 \alpha \tilde{P}+\tilde{Q}+\tilde{K}_{i}^{T} R \tilde{K}_{i}\right) \tilde{x}(t)\right. \\
& \left.+\tilde{\theta}^{T}(t) \tilde{P} \tilde{x}(t)+\tilde{x}^{T}(t) \tilde{P} \tilde{\theta}(t)\right)<0
\end{aligned}
$$

Afin d'obtenir les conditions LMI [23] qui permettent de vérifier [32], nous utilisons la notion d'ISS (Input to State Stability) (Sontag et Wang, 1995) en considérant des trajectoires bornées et variables dans le temps. Pour ce faire, on considère dans un premier temps le système [16] en régime autonome, c'est-à-dire 
$\tilde{\theta}(t)=0$ et l'état initial $\tilde{x}(0)$. D'après [32], nous pouvons déduire une fonction candidate de Lyapunov pour le système en régime autonome, notée $V_{\text {aut }}(x(t))$, et dont la dérivée s'exprime par :

$$
\begin{aligned}
\dot{V}_{\text {aut }}(x(t))= & \sum_{i=1}^{r} \sum_{j=1}^{r} h_{i}(z(t)) h_{j}(z(t)) \tilde{x}^{T}(t)\left(\tilde{A}_{i j}^{T} \tilde{P}+\tilde{P} \tilde{A}_{i j}\right. \\
& \left.+2 \alpha \tilde{P}+\tilde{Q}+\tilde{K}_{i}^{T} R \tilde{K}_{i}\right) \tilde{x}(t)<0
\end{aligned}
$$

Afin de garantir l'ISS de $\tilde{\theta}(t)$ sur $\tilde{x}(t)$, on majore [33] par deux fonctions $\delta(\|\tilde{\theta}(t)\|)$ et $\beta(\|\tilde{x}(t)\|)$ de classe $C^{\infty}$ telles que (Sontag et Wang, 1995):

$$
\dot{V}_{\text {aut }}(\tilde{x}(t)) \leq \beta(\|\tilde{\theta}(t)\|)-\delta(\|\tilde{x}(t)\|)
$$

A partir de [33], nous pouvons écrire :

$$
\dot{V}_{\text {aut }}(x(t))<-\lambda_{\min }\|\tilde{x}(t)\|^{2}
$$

où $\lambda_{\min }$ est la plus petite valeur propre de

$$
\sum_{i=1}^{r} \sum_{j=1}^{r} h_{i}(z(t)) h_{j}(z(t))\left(\tilde{A}_{i j}^{T} \tilde{P}+\tilde{P} \tilde{A}_{i j}+2 \alpha \tilde{P}+\tilde{Q}+\tilde{K}_{i}^{T} R \tilde{K}_{i}\right)
$$

Soit $\tilde{\theta}(t)=\tilde{\Gamma} r(t) \neq 0$ avec $\tilde{\Gamma}=\left[\begin{array}{lll}0 & -1 & 1\end{array}\right]^{T},[32]$ conduit à :

$$
\dot{V}(x(t)) \leq-\lambda_{\min }\|\tilde{x}(t)\|^{2}+r^{T}(t) \tilde{\Gamma}^{T}(t) \tilde{P} \tilde{x}(t)+\tilde{x}^{T}(t) \tilde{P} \tilde{\Gamma} r(t)
$$

Or $r^{T}(t) \tilde{\Gamma}^{T} \tilde{P} \tilde{x}(t)+\tilde{x}^{T}(t) \tilde{P} \tilde{\Gamma} r(t) \leq 2\left\|\tilde{X}^{T}(t) \tilde{P} \tilde{\Gamma} r(t)\right\| \leq 2\|\tilde{P} \tilde{\Gamma}\|\|\tilde{x}(t)\|\|r(t)\|$, d'où :

$$
\dot{V}(x(t)) \leq-\lambda_{\min }\|\tilde{X}(t)\|^{2}+2\|\tilde{P} \tilde{\Gamma}\|\|\tilde{x}(t)\|\|r(t)\|
$$

Notons que :

$$
\begin{aligned}
& -\frac{\lambda_{\min }}{2}\|\tilde{x}(t)\|^{2}+2\|\tilde{P} \tilde{\Gamma}\|\|\tilde{x}(t)\|\|r(t)\|-\frac{2}{\lambda_{\min }}\|\tilde{P} \tilde{\Gamma}\|^{2}\|r(t)\|^{2} \\
& =-\left(\sqrt{\frac{\lambda_{\min }}{2}}\|\tilde{x}(t)\|-\sqrt{\frac{2}{\lambda_{\min }}}\|\tilde{P} \tilde{\Gamma}\|\|r(t)\|\right)^{2} \leq 0
\end{aligned}
$$


En substituant [39] dans [38], on obtient :

$$
\begin{aligned}
\dot{V}(x(t)) & \leq-\lambda_{\text {min }}\|\tilde{x}(t)\|^{2}+2\|\tilde{P} \tilde{\Gamma}\|\|\tilde{x}(t)\|\|r(t)\| \\
& \leq-\frac{\lambda_{\text {min }}}{2}\|\tilde{x}(t)\|^{2}+\frac{2}{\lambda_{\text {min }}}\|\tilde{P} \tilde{\Gamma}\|^{2}\|r(t)\|^{2}
\end{aligned}
$$

Puisque $\|\tilde{P} \tilde{\Gamma}\|^{2}$ est borné, [40] assure que la propriété [34] est vraie et que l'ISS de [16] est garantie. D'après la propriété 1 , on peut écrire, pour $i, j=1, \ldots, r$ :

$$
\tilde{K}_{i}^{T} R \tilde{K}_{j} \leq \frac{1}{2}\left(\tilde{K}_{i}^{T} R \tilde{K}_{i}+\tilde{K}_{j}^{T} R \tilde{K}_{j}\right)
$$

Ainsi, l'inégalité [33] est vérifiée si :

$$
\sum_{i=1}^{r} \sum_{j=1}^{r} h_{i}(z(t)) h_{j}(z(t))\left(\tilde{A}_{i j}{ }^{T} \tilde{P}+\tilde{P} \tilde{A}_{i j}+2 \alpha \tilde{P}+\tilde{Q}+\frac{1}{2} \tilde{K}_{i}^{T} R \tilde{K}_{i}+\frac{1}{2} \tilde{K}_{j}{ }^{T} R \tilde{K}_{j}\right)<0
$$

L'inégalité [42] peut être réécrite sous sa forme développée avec les notations définies aux équations [16], [18], [28] et [31] telle que :

$$
\sum_{i=1}^{r} \sum_{j=1}^{r} h_{i}(z(t)) h_{j}(z(t)) \nabla_{i j}<0
$$

avec $\nabla_{i j}=\left[\begin{array}{ccccc}\nabla_{i j}(1,1) & (*) & (*) & (*) & (*) \\ \nabla_{i j}(2,1) & \nabla_{i j}(2,2) & (*) & (*) & (*) \\ \Delta A_{i}^{T} P_{1} & \left(A_{i}^{T}-A_{r}^{T}+\Delta A_{i}^{T}\right) P_{2} & A_{r}^{T} P_{3}+P_{3} A_{r}+2 \alpha P_{3} & 0 & 0 \\ -K_{i} & K_{i} & 0 & -2 R^{-1} & 0 \\ -K_{J} & K_{J} & 0 & 0 & -2 R^{-1}\end{array}\right]$

et $\nabla_{i j}(1,1)=P_{1}\left(A_{i}-L_{i} C_{j}\right)-P_{1} \Delta B_{i} K_{j}+(*)+2 \alpha P_{1}$,

$\nabla_{i j}(2,1)=-P_{2} B_{i} K_{j}-P_{2} \Delta B_{i} K_{j}+K_{j}^{T} \Delta B_{i}^{T} P_{1}+\Delta A_{i}^{T} P_{1}$,

$\nabla_{i j}(2,2)=P_{2}\left(A_{i}+\Delta A_{i}+B_{i} K_{j}+\Delta B_{i} K_{j}\right)+(*)+Q+2 \alpha P_{2}$.

On rappelle que $\Delta A_{i}(t)=H_{i} \Delta a(t) E_{a i} \quad$ et $\quad \Delta B_{i}(t)=H_{i} \Delta b(t) E_{b i} \quad$ avec $\Delta a^{T}(t) \Delta a(t) \leq I, \quad \Delta b^{T}(t) \Delta b(t) \leq I \quad$ et $\quad H_{i}, \quad E_{a i}, \quad E_{b i}, \quad E_{c i}$ des matrices à coefficients constants. D'après le lemme 1 , l'inégalité [43] est vérifiée si : 


$$
\sum_{i=1}^{r} \sum_{j=1}^{r} h_{i}(z(t)) h_{j}(z(t)) \mathbf{M}_{i j}<0
$$

$$
\text { Avec } \mathrm{M}_{i j}=\left[\begin{array}{ccccc}
\mathrm{M}_{i j}(1,1) & (*) & 0 & (*) & (*) \\
-P_{2} B_{i} K_{j} & \mathrm{M}_{i j}(2,2) & (*) & (*) & (*) \\
0 & \left(A_{i}^{T}-A_{r}^{T}\right) P_{2} & \mathrm{M}_{i j}(3,3) & 0 & 0 \\
-K_{i} & K_{i} & 0 & -2 R^{-1} & 0 \\
-K_{j} & K_{j} & 0 & 0 & -2 R^{-1}
\end{array}\right] \text {, }
$$

$$
\begin{aligned}
\mathrm{M}_{i j}(1,1) & =P_{1}\left(A_{i}-L_{i} C_{j}\right)+\left(A_{i}-L_{i} C_{j}\right)^{T} P_{1}+2 \alpha P_{1}+\left(\mu_{1}^{-1}+\mu_{5}{ }^{-1}\right) K_{j}^{T} E_{b i}{ }^{T} E_{b i} K_{j} \\
& +\left(\mu_{1}+\mu_{2}+\mu_{3}+\mu_{4}^{-1}\right) P_{1} H_{i} H_{i}^{T} P_{1}
\end{aligned}
$$

$$
\begin{aligned}
\mathrm{M}_{i j}(2,2) & =P_{2}\left(A_{i}+B_{i} K_{j}\right)+\left(A_{i}+B_{i} K_{j}\right)^{T} P_{2}+Q+2 \alpha P_{2}+\left(\mu_{2}^{-1}+\mu_{7}^{-1}\right) K_{j}{ }^{T} E_{b i}{ }^{T} E_{b i} K_{j}, \\
& +\left(\mu_{3}^{-1}+\mu_{6}{ }^{-1}\right) E_{a i}{ }^{T} E_{a i}+\left(\mu_{5}+\mu_{6}+\mu_{7}+\mu_{8}\right) P_{2} H_{i} H_{i}^{T} P_{2}
\end{aligned}
$$

et $\mathrm{M}_{i j}(3,3)=A_{r}^{T} P_{3}+P_{3} A_{r}+2 \alpha P_{3}+\left(\mu_{4}+\mu_{8}^{-1}\right) E_{a i}{ }^{T} E_{a i}$.

On pose $N=P_{2}^{-1}, \quad Y_{i}=K_{i} N$ et $Z_{i}=P_{1} L_{i}$, après congruence de [44] par $\operatorname{diag}\left[\begin{array}{lllll}N & N & I & I & I\end{array}\right]$, l'inégalité [43] est vérifiée si :

$$
\sum_{i=1}^{r} \sum_{j=1}^{r} h_{i}(z(t)) h_{j}(z(t)) \mathbb{S}_{i j}<0
$$

avec $\mathbb{S}_{i j}=\left[\begin{array}{c|cccc}\mathbb{S}_{i j}(1,1) & (*) & 0 & (*) & (*) \\ \hline-B_{i} N & \mathbb{S}_{i j}(2,2) & (*) & (*) & (*) \\ 0 & \left(A_{i}^{T}-A_{r}^{T}\right) & \mathbb{S}_{i j}(3,3) & 0 & 0 \\ -Y_{i} & Y_{i} & 0 & -2 R^{-1} & 0 \\ -Y_{j} & Y_{j} & 0 & 0 & -2 R^{-1}\end{array}\right]$,

$\mathbb{S}_{i j}(1,1)=N \Omega_{i j} N+\left(\mu_{1}^{-1}+\mu_{5}^{-1}\right) Y_{j}^{T} E_{b i}{ }^{T} E_{b i} Y_{j}$

où $\Omega_{i j}=P_{1} A_{i}+A_{i}^{T} P_{1}-Z_{i} C_{j}-C_{j}^{T} Z_{i}^{T}+2 \alpha P_{1}+\left(\mu_{1}+\mu_{2}+\mu_{3}+\mu_{4}{ }^{-1}\right) P_{1} H_{i} H_{i}^{T} P_{1}$,

$$
\begin{aligned}
\mathbb{S}_{i j}(2,2) & =A_{i} N+B_{i} Y_{j}+N A_{i}^{T}+Y^{T} B_{i}^{T}+N Q N+2 \alpha N+\left(\mu_{2}{ }^{-1}+\mu^{-1}\right) Y_{j}^{T} E_{b i}{ }^{T} E_{b i} Y_{j} \\
& +\left(\mu_{3}{ }^{-1}+\mu_{6}{ }^{-1}\right) N E_{a i}{ }^{T} E_{a i} N+\left(\mu_{5}+\mu_{6}+\mu_{7}+\mu_{8}\right) H_{i} H_{i}{ }^{T}
\end{aligned}
$$

et $\mathbb{S}_{i j}(3,3)=A_{r}^{T} P_{3}+P_{3} A_{r}+2 \alpha P_{3}+\left(\mu_{4}+\mu_{8}^{-1}\right) E_{a i}{ }^{T} E_{a i}$.

En appliquant le lemme 2, le bloc $\mathbb{S}_{i j}(1,1)$ de l'inégalité [45] peut être majoré, i.e. :

$$
N \Omega_{i j} N+\left(\mu_{1}^{-1}+\mu_{5}^{-1}\right) Y_{j}^{T} E_{b i}^{T} E_{b i} Y_{j} \leq-2 \varepsilon N-\varepsilon^{2} \Omega_{i j}^{-1}+\left(\mu_{1}^{-1}+\mu_{5}^{-1}\right) Y_{j}^{T} E_{b i}{ }^{T} E_{b i} Y_{j}
$$


Ensuite, en appliquant le complément de Schur, l'inégalité [45] est vérifiée si les conditions LMI [23] le sont.

Remarque 3: Notons que les conditions présentées au théorème précédent sont LMI avec un taux de décroissance $\alpha$ de la fonction de Lyapunov et les scalaires positifs $\mu_{1}, \mu_{2}, \mu_{3}$ et $\varepsilon$ fixés a priori.

\section{Exemple de simulation}

Afin d'illustrer l'approche proposée, on considère le problème de suivi de trajectoires d'un pendule inversé sur un chariot mobile décrit figure 4 .

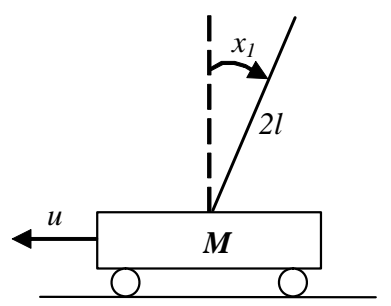

Figure 4 - Pendule inversé

Le modèle non linéaire du pendule est donné par (Wang et al., 1996) :

$$
\left\{\begin{array}{l}
\dot{x}_{1}(t)=x_{2}(t) \\
\dot{x}_{2}(t)=\frac{(m+M) g \sin \left(x_{1}(t)\right)-m l x_{2}^{2} \sin \left(x_{1}(t)\right) \cos \left(x_{1}(t)\right)-\cos \left(x_{1}(t)\right) u}{l\left(\frac{1}{3} m+\frac{4}{3} M+m \sin ^{2}\left(x_{1}(t)\right)\right)}
\end{array}\right.
$$

avec les paramètres donnés dans le tableau ci-dessous.

\begin{tabular}{ccc}
\hline Paramètres / Valeurs & Désignation & Unités \\
\hline$x_{1}(t)$ & Position angulaire & $\mathrm{rad}$ \\
$x_{2}(t)$ & Vitesse angulaire & $\mathrm{rad} / \mathrm{s}$ \\
$u(t)$ & Force appliquée sur le chariot & $\mathrm{N}$ \\
$g=9,8$ & Accélération de la pesanteur & $\mathrm{m} / \mathrm{s}^{2}$ \\
$m=0.1$ & Masse du pendule & $\mathrm{kg}$ \\
$M=1$ & Masse du chariot & $\mathrm{kg}$ \\
$2 l=1$ & Longueur du pendule & $\mathrm{m}$ \\
\hline
\end{tabular}

Tableau 1: Paramètres du pendule inversé 
Notons que le terme $m \sin ^{2}\left(x_{1}(t)\right)$ est négligeable devant $\frac{1}{3} m+\frac{4}{3} M$. De plus, on considère que le signal de vitesse $x_{2}(t)$ n'est pas mesurable. De ce fait, nous proposons de reporter la non linéarité $x_{2}^{2}(t)$ au sein des incertitudes paramétriques. Pour cela, nous supposons la vitesse bornée telle que $0 \leq x_{2}^{2}(t) \leq \bar{\eta}_{3}$ et le système [47] peut être approximé par le modèle non linéaire incertain :

$$
\left\{\begin{array}{l}
\dot{x}_{1}(t)=x_{2}(t) \\
\dot{x}_{2}(t)=d\left(x_{1}(t), u(t)\right)+\Delta d\left(x_{1}(t), x_{2}(t), u(t)\right)
\end{array}\right.
$$

avec $d\left(x_{1}(t), u(t)\right)=\frac{(m+M) g \eta_{1}\left(x_{1}(t)\right)-\eta_{2}\left(x_{1}(t)\right) u(t)}{\frac{1}{3}(4 M+m)}$,

$\Delta d\left(x_{1}(t), x_{2}(t), u(t)\right)=\frac{-m l \sin \left(x_{1}(t)\right) \cos \left(x_{1}(t)\right)}{\frac{l}{3}(4 M+m)} \bar{\eta}_{3} f\left(x_{2}(t)\right)$

$f\left(x_{2}(t)\right)=\frac{x_{2}^{2}(t)}{\bar{\eta}_{3}}$.

Afin d'obtenir un représentant TS de [48] par une décomposition en secteurs non linéaires, nous considérons les termes non linéaires $\sin \left(x_{1}(t)\right)$ et $\cos \left(x_{1}(t)\right)$. Soit $x_{1} \in\left[-\theta_{0}, \theta_{0}\right]$, d'après le lemme 1 , on peut écrire :

$$
\begin{aligned}
& \sin \left(x_{1}(t)\right)=\omega_{1}^{1}\left(x_{1}(t)\right) x_{1}(t)+\omega_{1}^{2}\left(x_{1}(t)\right) \frac{\sin \left(\theta_{0}\right)}{\theta_{0}} x_{1}(t) \\
& \cos \left(x_{1}(t)\right)=\omega_{2}^{1}\left(x_{1}(t)\right)+\omega_{2}^{2}\left(x_{1}(t)\right) \cos \left(\theta_{0}\right)
\end{aligned}
$$

avec $\omega_{1}^{1}\left(x_{1}(t)\right)=\frac{\theta_{0} \sin \left(x_{1}(t)\right)-x_{1}(t) \sin \left(\theta_{0}\right)}{x_{1}(t)\left(\theta_{0}-\sin \left(\theta_{0}\right)\right)}, \omega_{1}^{2}\left(x_{1}(t)\right)=1-\omega_{1}^{1}\left(x_{1}(t)\right)$,

$\omega_{2}^{1}\left(x_{1}(t)\right)=\frac{\cos \left(x_{1}(t)\right)-\cos \left(\theta_{0}\right)}{1-\cos \left(\theta_{0}\right)}$ et $\omega_{2}^{2}\left(x_{1}(t)\right)=1-\omega_{2}^{1}\left(x_{1}(t)\right)$.

Une manière de réduire le conservatisme des conditions de LMI est de réduire le nombre de règles employées pour modéliser le système (Taniguchi et al., 2001). Avec le découpage des non linéarités [49] et [50], le modèle TS obtenu devrait comporter 4 règles. Néanmoins, notons que $\omega_{1}^{1}$ et $\omega_{2}^{1}$, respectivement $\omega_{1}^{2}$ et $\omega_{2}^{2}$, sont 
superposées (Guerra et al., 2001). De ce fait, un représentant TS à deux règles du système incertain [48] peut être obtenu. Celui-ci est donné par :

$$
\left\{\begin{array}{l}
\dot{x}(t)=\sum_{i=1}^{2} h_{i}\left(x_{1}(t)\right)\left(\left(A_{i}+\Delta A_{i}(x(t))\right) x(t)+B_{i} u(t)\right) \\
y(t)=C x(t)
\end{array}\right.
$$

$\operatorname{avec} x(t)=\left[\begin{array}{ll}x_{1}(t) & x_{2}(t)\end{array}\right]^{T}, A_{1}=\left[\begin{array}{cc}0 & 1 \\ \frac{(M+m) g}{\frac{l}{3}(4 M+m)} & 0\end{array}\right]$,

$A_{2}=\left[\begin{array}{cc}0 & 1 \\ \frac{(M+m) g}{\frac{l}{3}(4 M+m)} \frac{\sin \left(\theta_{0}\right)}{\theta_{0}} & 0\end{array}\right], \Delta A_{1}(t)=H_{1} \Delta_{a}(t) E_{a 1}, \Delta A_{2}(t)=H_{2} \Delta_{a}(t) E_{a 2}$,

$H_{1}=\left[\begin{array}{c}0 \\ \frac{-m l}{\frac{l}{3}(4 M+m)}\end{array}\right], E_{a 1}=\left[\begin{array}{ll}\bar{\eta}_{3} & 0\end{array}\right], H_{2}=\left[\begin{array}{c}0 \\ \frac{-m l}{\frac{l}{3}(4 M+m)} \frac{\sin \left(\theta_{0}\right) \cos \left(\theta_{0}\right)}{\theta_{0}}\end{array}\right]$,

$E_{a 2}=\left[\begin{array}{ll}\bar{\eta}_{3} & 0\end{array}\right], \quad B_{1}=\left[\begin{array}{c}0 \\ -\frac{1}{\frac{l}{3}(4 M+m)}\end{array}\right], \quad B_{2}=\left[\begin{array}{c}0 \\ -\frac{\cos \left(\theta_{0}\right)}{\frac{l}{3}(4 M+m)}\end{array}\right], \quad C=\left[\begin{array}{ll}1 & 0\end{array}\right] \quad$ et les

fonctions d'appartenance $h_{1}\left(x_{1}(t)\right)=\omega_{1}^{1}\left(x_{1}(t)\right)$ et $h_{2}\left(x_{1}(t)\right)=\omega_{1}^{2}\left(x_{1}(t)\right)$.

Afin de réaliser la synthèse du correcteur, on considère une vitesse angulaire maximale $\bar{x}_{2}=0.1 \mathrm{rad} / \mathrm{s}^{2}$, c'est-à-dire $\bar{\eta}_{3}=0.01$. De plus, notons que le système est non commandable en $x_{1}= \pm \pi / 2$. De ce fait, nous avons choisi de travailler dans le sous-espace compact défini par $x_{1} \in\left[-\theta_{0}, \theta_{0}\right]$ avec $\theta_{0}=22 \pi / 45 \mathrm{rad}$.

Les solutions du théorème ont été obtenues pour une valeur minimale de la fonction de coût $\lambda=0.0225$ avec la dynamique du modèle de référence choisie telle que $A_{r}=\left[\begin{array}{cc}0 & 1 \\ -6 & -5\end{array}\right]$, un taux de décroissance $\alpha=1.9$ et les conditions initiales $x^{T}(0)=\left[\begin{array}{ll}0.2 & 0\end{array}\right]$ et $\hat{x}^{T}(0)=\left[\begin{array}{ll}0 & 0.1\end{array}\right]$. Les gains $K_{i}$ de la loi de commande et $L_{i}$ de l'observateur, solutions du théorème 1 obtenue via la LMI Toolbox de Matlab (Gahinet et al., 1995), sont donnés par :

$$
\begin{aligned}
& K_{1}=\left[\begin{array}{ll}
577.4841 & 253.4788
\end{array}\right], K_{2}=\left[\begin{array}{ll}
577.6043 & 253.5295
\end{array}\right], \\
& L_{1}{ }^{T}=\left[\begin{array}{ll}
20 & 210.2651
\end{array}\right], L_{2}{ }^{T}=\left[\begin{array}{ll}
20 & 215.7756
\end{array}\right],
\end{aligned}
$$


De plus, les scalaires et matrices suivants complètent la solution :

$$
\begin{aligned}
& \varepsilon=100, \mu_{1}=0.01, \mu_{2}=0.01, \mu_{3}=100, \mu_{4}=0.2332 .10^{-7}, \\
& \mu_{5}=0,4772.10^{6}, \mu_{6}=0,4772.10^{6} \quad \mu_{7}=0,4772.10^{6}, \mu_{8}=0,4772.10^{6}, \\
& P_{1}=\left[\begin{array}{cc}
0.5294 & -0.0460 \\
-0.0460 & 0.0061
\end{array}\right], N=10^{5} .\left[\begin{array}{cc}
0.5919 & -1.3398 \\
-1.3398 & 3.0468
\end{array}\right], \\
& P_{3}=10^{7} \cdot\left[\begin{array}{ll}
5.5519 & 2.2863 \\
2.2863 & 1.0113
\end{array}\right], Q=\left[\begin{array}{ll}
0.2324 & 0.0004 \\
0.0004 & 0.2315
\end{array}\right], R=5.10^{-6} .
\end{aligned}
$$

Les figures (5-10) illustrent les simulations effectuées pour une poursuite de trajectoire imposée par le signal de référence $r(t)=\left[\begin{array}{c}0 \\ 2.46 \sin (t)\end{array}\right]$. Les figures (58) illustrent la convergence de l'état vers la trajectoire désirée ainsi que celle de l'état estimé par l'observateur. Le signal de commande et l'erreur quadratique sont donnés respectivement sur les figures 9 et 10 .

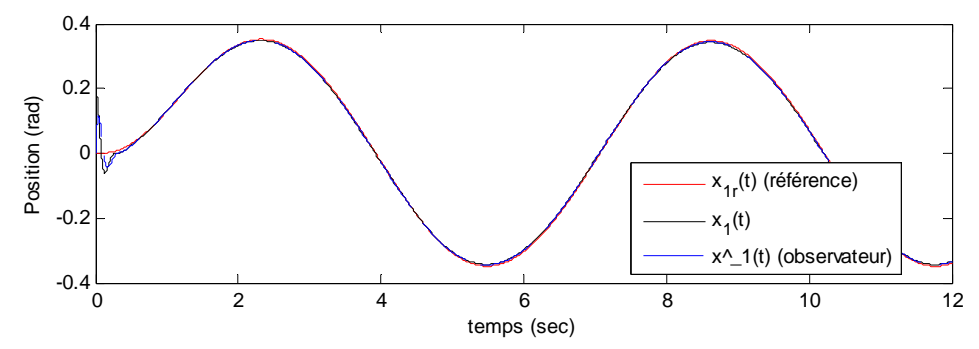

Figure 5 - Poursuite en position.

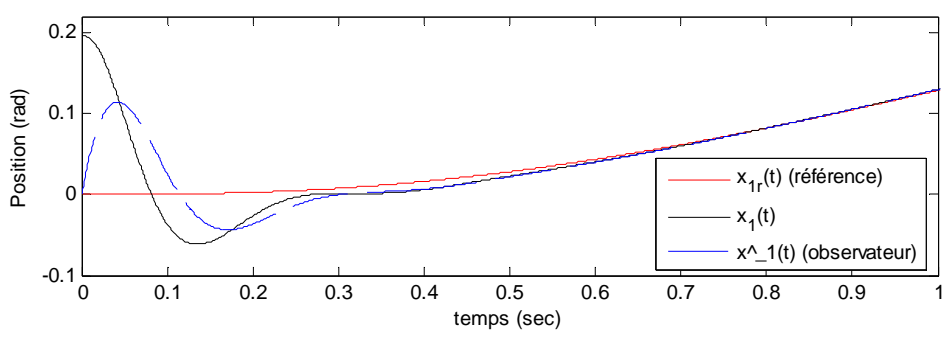

Figure 6 - Poursuite en position (Zoom). 


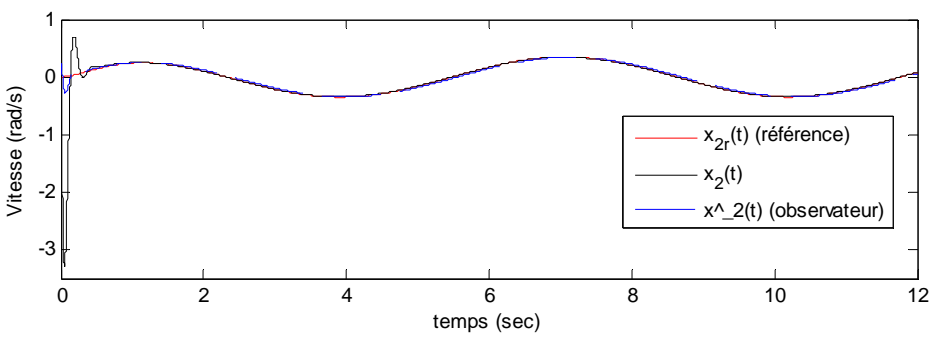

Figure 7 - Poursuite en vitesse

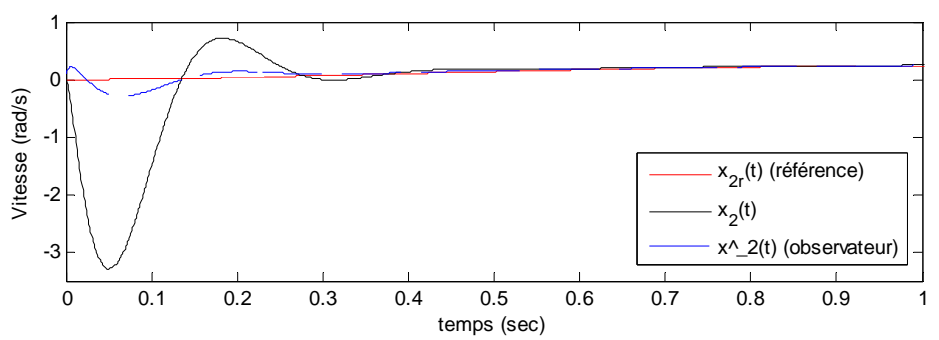

Figure 8 - Poursuite en vitesse (Zoom)

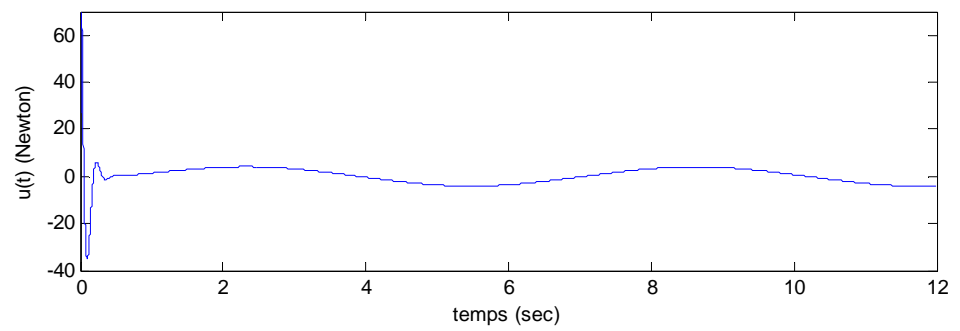

Figure 9 - Signal de commande

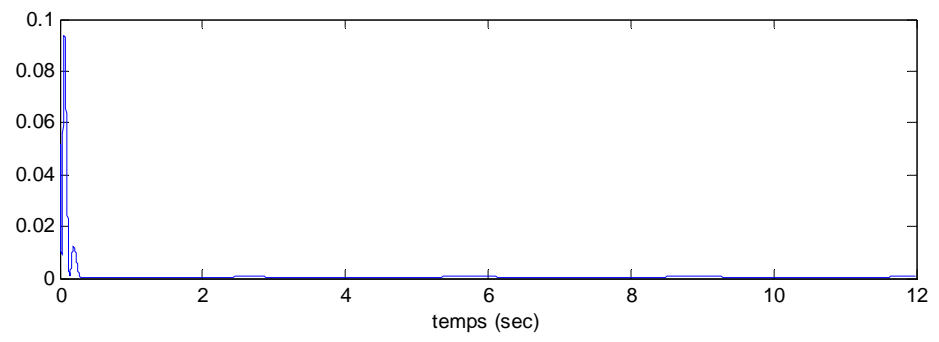

Figure 10 - Erreur quadratique de poursuite 


\section{Conclusion}

Dans cet article, nous avons proposé des conditions de stabilisation LMI pour la poursuite de trajectoires des modèles non linéaires incertains représentés sous la forme quasi-LPV (modèles TS). La structure de commande par retour de sortie considérée repose sur l'introduction d'un modèle de référence et d'un observateur TS. A partir de la minimisation d'une fonction de coût quadratique relative à l'erreur de poursuite, des conditions de synthèse sous optimale des gains d'observation et de commande ont été obtenues par le biais des propriétés de l'ISS (Input to State Stability). L'efficacité de l'approche de synthèse proposée a été illustrée en simulation pour l'exemple classique d'un pendule inversé mis en mouvement par un chariot mobile.

\section{Bibliographie}

Alazard D., Cumer C., Apkarian P., Gauvrit M., Ferreres G., Robustesse et Commande Optimale, Cepaduès, 1999.

Assawinchaichote W., Nguang S.K., Shi P., " $H_{\infty}$ output feedback control design for uncertain singularly perturbed systems: an LMI approach", Automatica, vol. 40, n ${ }^{\circ} 12$, 2004, p. 2147-2152.

Ban X., Gao X.Z., Huang X., Vasilakos A.V., "Stability analysis of the simplest TakagiSugeno fuzzy control system using circle criterion", Information Sciences (2007), vol. $177, \mathrm{n}^{\circ} 20,2007$, p. $4387-4409$.

Bouarar T., Guelton K., Manamanni N., "Static output feedback controller design for TakagiSugeno systems - A fuzzy Lyapunov LMI approach", 48th IEEE Conference on Decision and Control CDC'09, Shanghai, P.R. China, 16-18 décembre 2009.

Boyd S., El Ghaoui L., Feron E., Balakrishnan V., Linear Matrix Inequalities in System and Control Theory, Philadelphia, PA: SIAM, 1994.

Cao Y.Y., Frank P.M., "Stability analysis of nonlinear time-delay systems via linear TakagiSugeno fuzzy models", Fuzzy Sets \& Systems vol. 124, 2001, p. 213-229.

Chadli M., El Hajjaji A., "Comment on Observer-based robust fuzzy control of nonlinear systems with parametric uncertainties", Fuzzy Sets \& Systems, vol. 157, n 9 , 2006, p. $1276-1281$.

Chadli M., Maquin D., Ragot J., "An LMI formulation for output feedback stabilisation in multiple model approach", 41th IEEE Conference on Decision Control CDC'02, USA, 10-13 décembre 2002.

Chen B.S., Tseng C.S., Uang H.J., "Mixed $\mathrm{H}_{2} / \mathrm{H}_{\infty}$ fuzzy output feedback control design for nonlinear dynamic systems: an LMI approach", IEEE Trans. on Fuzzy Systems, vol. 8, n³, 2000, p. 249-265.

Gahinet P., Nemirovski A., Laub A.J., Chilali M., LMI control toolbox. The Mathworks, Inc., 1995. 
Gasso K., Mourot G., Boukhriss A., Ragot J., « Optimisation de la structure d'un modèle de Takagi-Sugeno », Actes des rencontres francophones sur la logique floue LFA'99, Valenciennes, France, 1999, Cépadues.

Gasso K., Identification des systèmes dynamiques non-lneaires : approche multi-modèles, Thèse de doctorat, INPL, 2000

Guelton K., Bouarar T., Manamanni N., "Robust dynamic output feedback fuzzy Lyapunov stabilization of Takagi-Sugeno systems - A descriptor redundancy approach", Fuzzy Sets and Systems, vol. 160, n 19,2009 , p. 2796-2811.

Guerra T.M., Vermeiren L., "Stabilité et stabilisation à partir de modèles flous », in: L. Foulloy, S. Galichet and A. Titli (eds), Commande Floue 1: de la stabilisation à la supervision, Traités IC2 Systèmes Automatisés, Hermes Lavoisier,Paris, 2001, p. 59-98.

Guerra T.M., Kruszewski A., Vermeiren L., Tirmant H., "Conditions of output stabilization for nonlinear models in the Takagi-Sugeno's form”, Fuzzy Set and Systems, vol. 157, 2006, p. 1248-1259.

Huang D., Nguang S.K., "Static output feedback controller design for fuzzy systems: An ILMI approach”, Information Sciences, vol. 177, n¹4, 2007, p.3005-3015.

Hong S.K., Langari R., “An LMI-based fuzzy control system design with TS framework”, Information Sciences, vol. 123, 2000, p. 163-179.

Li J., Wang H.O., Newmann D., Tanaka K., "Dynamic parallel distributed compensation For Takagi Sugeno systems: An LMI approach”, Information Sciences, vol. 123, 2000, p. 201-221.

Liu X., Zhang Q., "New approaches to Ho controller design based on fuzzy observers for fuzzy T-S systems via LMI”, Automatica, vol. 39, n9, 2003, p. 1571-1582.

Ma X.J., Sun Z.Q., He Y.Y., “Analysis and design of fuzzy controller and fuzzy observer”, IEEE Trans. on Fuzzy Systems, vol. 6, n 1, 1998, p. 41-50.

Mansouri B., Kruszewski A., Guelton K., Manamanni N., "Sub-optimal output tracking control design for uncertain T-S models", $3^{\text {rd }}$ IFAC Workshop on Advanced Fuzzy and Neural Control, Valenciennes, France, vol. 3, n¹, 2007, p. 91-96.

Mansouri B., Manamanni N., Guelton K., Kruszewski A., Guerra T.M., “Output feedback LMI tracking control conditions with $\mathrm{H} \infty$ criterion for uncertain and disturbed TS models", Information Sciences, vol. 179, n4, 2009, p. 446-457.

Morère Y., Mise en oeuvre de lois de commande pour les modèles flous de type TakagiSugeno, Thèse de doctorat, Université de Valenciennes et du Hainaut-Cambrésis, 2001.

Park W., "LMI-based robust stability analysis for fuzzy feedback linearization regulators with its applications", Information Sciences, vol. 152, 2003, p. 287-301.

Park Y., Tahk M., Bang H., "Design and analysis of optimal controller for fuzzy systems with input constraint", IEEE Transactions on Fuzzy Systems, vol 12, n 6.2004, p. 766-779.

Sala A., Guerra T.M., Babuska R., "Perspectives of fuzzy systems and control”, Fuzzy Sets and Systems, vol. 156, n³, 2005, p. 432-444. 
24 RS - JESA. Volume $\mathrm{X}-\mathrm{n}^{\circ} \mathrm{x} /$ année. Commande robuste et applications

Sontag, E.D., Wang Y., "On characterizations of the input-to-state stability property", System and Control Letters, vol. 24, 1995, p. 351-359.

Syrmos V.L., Abdallah C.T., Dorato P., Grigoriadis K., "Static output feedback - A survey", Automatica, vol. 33, n², 1997, p. 125-137.

Takagi T., Sugeno M., "Fuzzy identification of systems and its application to modelling and control", IEEE Trans. Syst., Man and Cyber, vol. 1115, 1985, p. 116-132.

Tanaka K., Wang. H.O., Fuzzy control systems design and analysis : a linear matrix inequality approach, John Wiley \& Son Eds, New York, Wiley-Interscience, 2001.

Tanaka K., Ikeda T., Wang H.O., "Robust Stabilzation of a Class of Uncertain Nonlinear Systems via Fuzzy Control: Quadratic Stabilizability, Ho Control Theory, and Linear Matrix Inequalities”, IEEE Trans. Fuzzy Sys., vol. 4, n 1, 1996.

Tanaka K., Ikeda T., Wang H.O., "Fuzzy regulators and fuzzy observers: Relaxed stability conditions and LMI-based designs", IEEE Trans. Fuzzy Sys., vol. 6, n², 1998.

Taniguchi T., Tanaka K., Wang H.O., "Model construction rule reduction and robust compensation for generalized form of Takagi-Sugeno fuzzy systems", IEEE Trans. on Fuzzy Systems, vol. 9, n4, 2001, p. 525-537.

Tong S., Wang T., Li H.X., "Fuzzy robust tracking control for uncertain nonlinear systems", International Journal of Approximate Reasoning, vol. 30, 2002, p. 73-90.

Tseng C., Chen B., "Hळ decentralized fuzzy model reference tracking control design for non linear interconnected systems”, IEEE Trans., Fuzzy, Sys., vol. 9, n 6, 2001.

Tseng C., Chen B., Uang H.J., "Fuzzy tracking Control Design for nonlinear Dynamic systems via T-S fuzzy model”, IEEE Trans., Fuzzy, Sys., vol. 9, n 3, 2001, p. 381-392.

Wang H.O., Tanaka K., Griffin M., "An approach to fuzzy control of non linear systems : Stability and design issues", IEEE Transactions on Fuzzy Systems, vol. 4, 1996, p. 14-23.

Wu S.J., Lin C.T., "Optimal fuzzy controller design in continuous fuzzy system: global concept approach", IEEE Transactions on Fuzzy Systems, vol. 8, n 6., 2000, p. 713-729.

$\mathrm{Xu}, \mathrm{S}$., Lam J., "Robust H-infinity control for uncertain discrete-time-delay fuzzy systems via output feedback controllers", IEEE Trans. on Fuzzy Sys., vol. 13, n¹, 2005, p. 82-93.

Yoneyama J., Nishikawa M., Katayama H., Ichikawa A., "Output stabilization of TakagiSugeno fuzzy systems”, Fuzzy Sets and Systems, vol. 111, 2000, p. 253-266.

Zerar M., Guelton K., Manamanni N., "Linear Fractional Transformation based H-infinity output stabilization for Takagi-Sugeno fuzzy models", Mediterranean Journal of Measurement and Control, vol. 4, n³, 2008, p. 111-121.

Zhou K., Khargonedkar P., "Robust Stabilization of linear systems with norm-bounded timevarying uncertainty", Sys. Control Letters, vol. 10, 1988, p. 17-20. 